\title{
Subsectional Tapered Fed Printed LPDA Antenna With a Feeding Point Patch
}

\author{
Goksenin Bozdag and Alp Kustepeli
}

\begin{abstract}
In this letter, a log-periodic dipole array (LPDA) antenna operating between 1.1 and $13.8 \mathrm{GHz}$ with VSWR $<2$ and an average peak realized gain of $5.2 \mathrm{~dB}$ is presented. It is suitable for GPS (L1-L5), PCS, IMT-2000, Bluetooth, WLAN, WiMAX, UWB, and $X$-band applications. The antenna is designed by introducing a subsectional tapered feedline instead of standard microstrip feeding, which leads to a remarkable lower-frequency performance. The upper-frequency performance of the antenna is improved by adding a feeding point patch. The group delay is also examined for the final design, and maximum $1.5 \mathrm{~ns}$ deviation is observed in UWB. By using ordinary and simple dipoles with this modified feedline approach, a superior antenna performance is obtained over a very wide frequency band, and it is validated by the comparison of three fabricated antennas. Additionally, the antenna size is also reduced without using complicated and/or top-loaded dipole structures.
\end{abstract}

Index Terms-Feeding point patch, printed log-periodic dipole array antenna, subsectional tapered feeding.

\section{INTRODUCTION}

$\mathbf{L}$ OG-PERIODIC dipole array (LPDA) antenna achieves high directivity in a very wide frequency range. Therefore, it is indispensable and it has never lost its importance since its first introduction [1]. It is widely used in many different applications both in industry and military [2]-[9]. In today's spaceand weight-limited applications, antennas need to be wideband, compact, lightweight, relatively small, and planar for easy fabrication and integration. Using only one device for many communication systems such as GPS, PCS, IMT-2000, Bluetooth, WLAN, WiMAX, and UWB is also desired [10], [11]. Additionally, utilization of X-band used for various radar applications and its combination with the systems above are preferred to improve the functionality of mobile devices [12]. Frequency designations are given in Table I for convenience. Printed LPDA (PLPDA) antennas can be used for those purposes, and there are many studies examining PLPDAs in the literature. However, it is not easy to obtain an antenna satisfying the desired specifications because impedance mismatches easily deteriorate the

Manuscript received April 18, 2015; revised May 20, 2015; accepted June 28, 2015. Date of publication June 30, 2015; date of current version February 26, 2016.

The authors are with the Department of Electrical and Electronics Engineering, Izmir Institute of Technology, Izmir 35430, Turkey (e-mail: gokseninbozdag@iyte.edu.tr).

Color versions of one or more of the figures in this letter are available online at http://ieeexplore.ieee.org.

Digital Object Identifier 10.1109/LAWP.2015.2451395
TABLE I

FREQUENCY ALLOCATIONS

\begin{tabular}{c|cc}
\hline \hline \multicolumn{2}{c}{ Band Designation } & \multicolumn{2}{c}{ Frequency (GHz) } \\
\hline & 1.176 & (L5) \\
Global Positioning System & 1.379 & (L2) \\
(GPS) & 1.381 & (L4) \\
& 1.575 & (L3) \\
\hline Public Communication & $1.710-1.784$ & (uplink) \\
System (PCS) & $1.805-1.879$ & (downlink) \\
\hline IMT-2000 & $2.110-2.200$ & (preference) \\
\hline Bluetooth & $2.400-2.485$ & $(802.15 .1)$ \\
\hline Wireless Local Area & $2.412-2.472$ & $(802.11 / \mathrm{a}-\mathrm{n})$ \\
Network (WLAN) & $3.657-3.690$ & $(802.11 \mathrm{y})$ \\
& $4.942-4.948$ & $(802.11 / \mathrm{a}-\mathrm{j})$ \\
& $5.180-5.825$ & (802.11/a-n) \\
\hline WiMAX & $2.4,5.8$ & (unlicensed) \\
& $2.5,3.5,10.5$ & (licensed) \\
\hline Ultra Wide Band (UWB) & $3.1-10.6$ & (802.15.3) \\
\hline X-band & $8.0-12.0$ & \\
\hline \multicolumn{2}{c}{}
\end{tabular}

wideband characteristics. For their effective usage, their bandwidth performances need to be enhanced and their sizes must be reduced. Although employing complicated dipole structures and different radiators provides about $30 \%$ size reduction, the bandwidths of the antennas are not satisfactory for wideband applications [4], [6], [7], [9]. In [7], a PLPDA with 16 complicated and top-loaded elements was proposed, and it merely covers the bandwidth from 0.87 to $7.42 \mathrm{GHz}$ even for an input reflection coefficient $\left|S_{11}\right|<-6.5 \mathrm{~dB}$ (VSWR $<2.8$ ), which is actually a high value, and the average measured gain of the antenna is about $2 \mathrm{~dB}$. If one considers the general bandwidth requirement $\left|S_{11}\right|<-10 \mathrm{~dB}(\mathrm{VSWR}<2)$, the bandwidth is poorly degraded. Similar interpretations can be made for [4], [10], and [13] where the bandwidth is designated according to $\left|S_{11}\right|<-6 \mathrm{~dB}(\mathrm{VSWR}<3)$ and [9], as well.

In regard to antenna feeding, two very well-known structures, coaxial cable and standard microstrip lines with constant widths including the coplanar one, are used. In fact coaxial feeding is not very suitable for printed structures since it requires additional realization efforts and deforms the planar form [3], [7]-[9]. It is also seen that the standard microstrip feedings do not improve the bandwidth when compared with the coaxial one [5], [8]. Moreover, patches or short tapers at the input that are between the feed point and the array might be used as impedance transitions. However, they do not make significant contributions in terms of bandwidth ratio [2], [3], [8], [13], [14]. In [14], the antenna with the dimensions of a standard PLPDA, consisting of 12 elements and a short taper transition at the input, only covers the frequency range between 


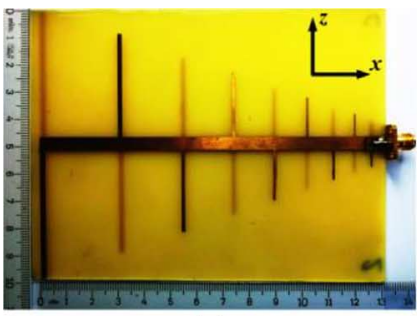

(a)

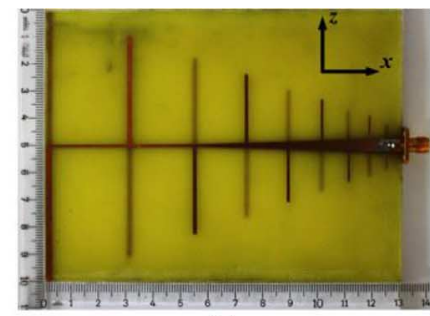

(b)

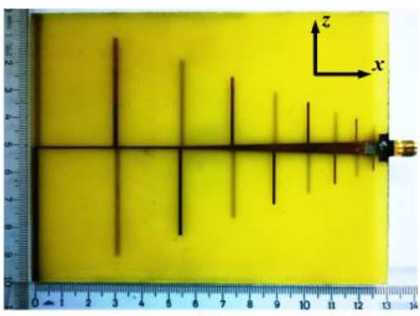

(c)

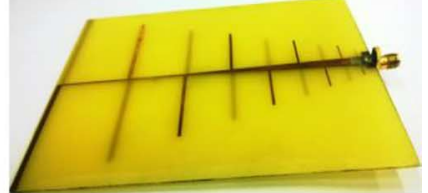

(d)

Fig. 1. PLPDA antennas. (a) Basic, (b) SsTF, (c) SsTFwP, and (d) top view of SsTFwP.

TABLE II

GeOMETRICAL PARAMETERS OF LPDAs

\begin{tabular}{c|ccccccccc}
\hline \hline$n$ & 1 & 2 & 3 & 4 & 5 & 6 & 7 & 8 & 9 \\
\hline$l_{n}(\mathrm{~mm})$ & 100 & 80 & 64 & 51.2 & 41 & 32.8 & 26.2 & 21 & 16.8 \\
\hline$w_{n}(\mathrm{~mm})$ & 1.8 & 1.4 & 1.1 & 0.91 & 0.73 & 0.59 & 0.47 & 0.38 & 0.3 \\
\hline$d_{n}(\mathrm{~mm})$ & 29.8 & 23.8 & 19.1 & 15.3 & 12.2 & 9.8 & 7.8 & 6.2 & - \\
\hline \hline
\end{tabular}

1 and $4 \mathrm{GHz}(4: 1)$ and it is not actually suitable for wideband applications. Therefore, in this study, the whole feeding line modifications are investigated to improve the wideband characteristics of PLPDA antennas. Preliminary comprehensive numerical studies are performed by using HFSS to obtain the optimum design before the fabrications and measurements.

\section{ANTENNA Designs, Results, AND COMPARISONS}

General configuration of LPDA antenna is described by the scaling factor $\tau=R_{n+1} / R_{n}=l_{n+1} / l_{n}=w_{n+1} / w_{n}=$ $d_{n+1} / d_{n}$, where $R_{n}$ is the distance between the $n$th element and the apex, and $l_{n}$ and $w_{n}$ are the length and the width of the $n$th element, respectively. The element spacing $d_{n}=R_{n}$ $R_{n+1}$, the spacing factor $\sigma=d_{n} /\left(2 l_{n}\right)$, and the half-angle $\alpha=\tan ^{-1}[(1-\tau) /(4 \sigma)]$ [15]. Additionally, a set of contours related to these parameters are used to determine the optimum directivity [15].

In this study, it is intended to design a PLPDA antenna with VSWR $<2$ and a gain of $7 \mathrm{~dB}$ to cover the frequency range from 1 to $12 \mathrm{GHz}$ for the bands mentioned in Section I. The basic parameters $\tau, \sigma$, and $\alpha$ are determined as $0.8,0.149$, and $18.56^{\circ}$, respectively. The lengths of the strips are basically related with the effective dielectric constant $\varepsilon_{\mathrm{e}}$ of substrate and the length of the longest strip $l_{1}=\lambda_{\max } /\left(2 \sqrt{\varepsilon_{\mathrm{e}}}\right)$, where $\lambda_{\max }$ is the maximum wavelength in the band. The widths of the strips are chosen as $w_{n}=\pi a_{n}$ [16], where $a_{n}$ is the diameter of the corresponding wire element determined according to the procedure described in [15] and [16]. The lengths and widths of the other strips are determined by using $\tau$ with $l_{1}$ and $w_{1}$. The antennas were fabricated using photolithography on FR4 whose thickness, dielectric constant, and tangent loss are $1.5 \mathrm{~mm}, 4.5$, and 0.02 , respectively, and $\varepsilon_{\mathrm{e}}$ was determined as 2.25 by the comparisons of preliminary computational and experimental results. Although the total number of elements is calculated as 16 according to the general design procedure for a standard LPDA antenna [15], in the preliminary computational analysis, it was also seen that the desired bandwidth could be obtained by using only the first 9 elements. Since the accurate realization of very

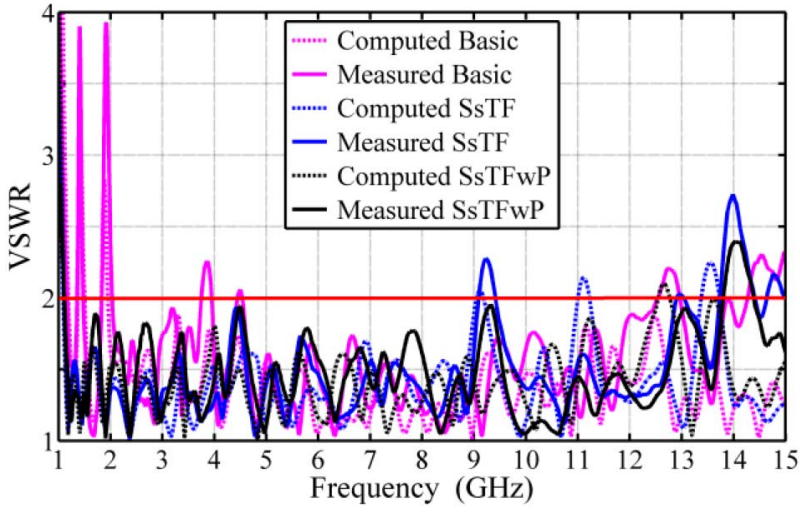

Fig. 2. VSWR of PLPDA antennas.

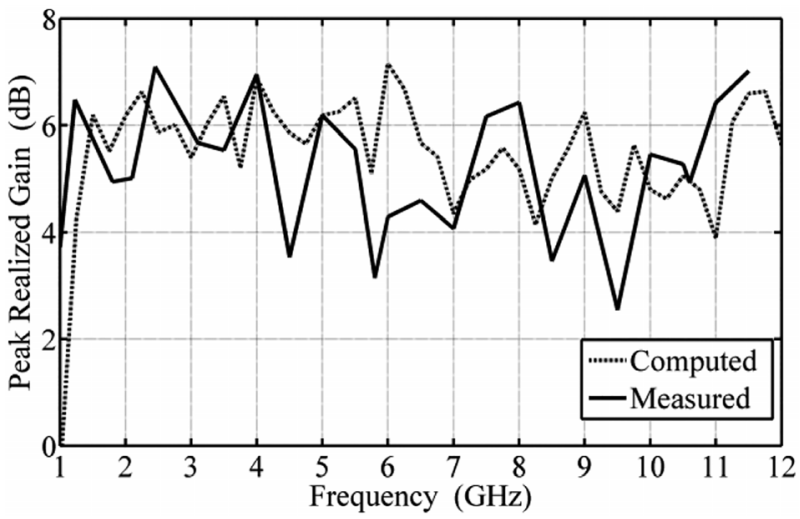

Fig. 3. Peak realized gain of SsTFwP antenna.

narrow strips resulting from the usage of high number of elements is very difficult, it is preferred to use as few elements as possible. The length of the antenna is optimized as $130 \mathrm{~mm}$. The width of the antenna is determined by the length of the first and the longest element $l_{1}$, which is $100 \mathrm{~mm}$, and its width $w_{1}$ is obtained as $1.8 \mathrm{~mm}$. The lengths and the widths of the elements and the spacings between them are given in Table II.

These dimensions are similar to those of the antenna consisting of 16 triangular and top-loaded elements with the dimensions of $88 \mathrm{~mm} \times 135 \mathrm{~mm}$, presented in [7], which was obtained as the miniaturized form for a standard PLPDA with 16 elements and the dimensions of $130 \mathrm{~mm} \times 244 \mathrm{~mm}$. As a result, the proposed design can also be considered as the reduced form of that standard PLPDA. The width of the feeding line is chosen as $4.5 \mathrm{~mm}$ to obtain a $50-\Omega$ characteristic line impedance, which is very close to the one with $52 \Omega$ obtained according to [15]. 

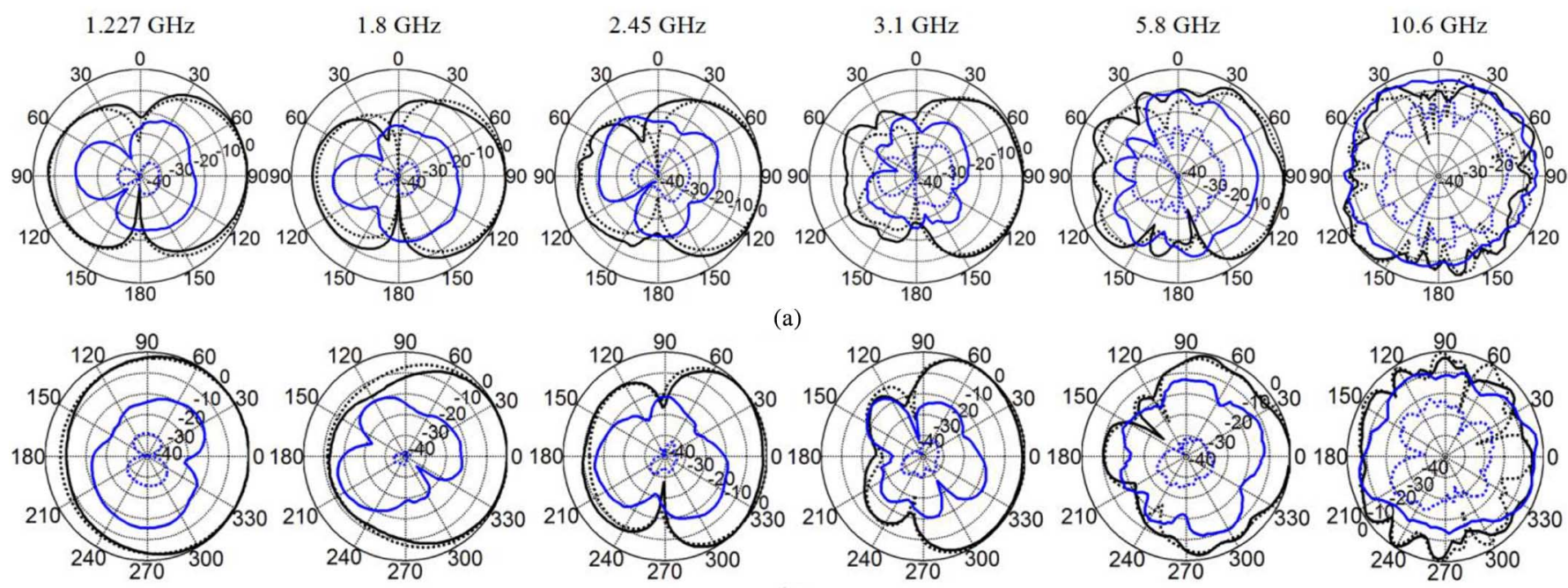

(a)
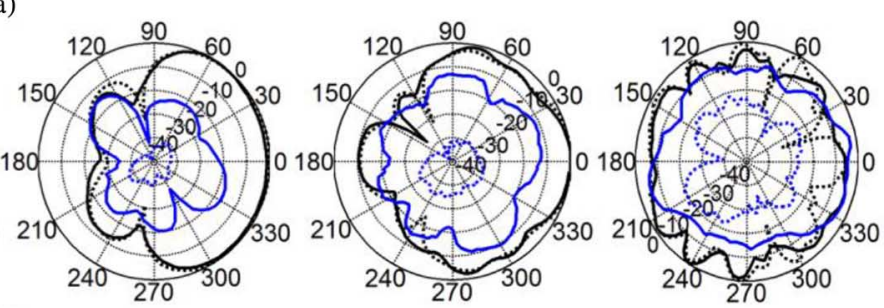

(b)

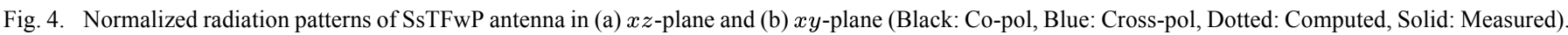

The resulting antenna is called a basic PLPDA antenna. The fabricated antennas and their computed and measured VSWR values are shown in Figs. 1 and 2. The bandwidth performance of the basic PLPDA antenna shown in Fig. 1(a) seems to be satisfactory above $2 \mathrm{GHz}$ according to the computational results in Fig. 2. However, it is very poor below $2 \mathrm{GHz}$, where GPS and PCS are allocated. In the measured results, it is seen that the bandwidth deteriorations do not only occur below $2 \mathrm{GHz}$, but they also occur between 3.75 and $4.5 \mathrm{GHz}$. The upper operating frequency limit of the antenna is $12.5 \mathrm{GHz}$. It is obvious that the basic PLPDA antenna has two main operating bands, which are 2-3.75 and 4.5-12.5 GHz. As a result, the basic PLPDA antenna is not sufficient for wideband applications and multipurpose devices in the specified bandwidth.

To improve its performance while keeping the dimensions and the number of elements the same, the modification of the feedline is proposed in the second phase of the study. A subsectional tapered feeding line is used instead of the whole feeding line with constant width. The proposed feeding line includes two different tapered subsections, and both of them are designed and optimized specifically to get a smooth impedance matching. Therefore, the resulting antenna is called subsectional tapered fed (SsTF) PLPDA antenna. The widths of the tapered lines increase toward the feeding port, while the lengths of elements decrease in the same direction as seen in Fig. 1(b). The length of the first subsection is $55.2 \mathrm{~mm}$, and its width increases from 0.5 to $0.7 \mathrm{~mm}$, from the longest dipole to the third longest dipole. The second subsection is longer and wider than the first one. Its length is $74.8 \mathrm{~mm}$, and its width increases from 0.7 to $4.5 \mathrm{~mm}$, from the third longest dipole to the feeding port.

In the preliminary numerical analysis, the feed structures obtained by using only one tapered line or by setting the tapered line in the other direction were also investigated, but the results were not satisfactory according to the bandwidth requirement determined as VSWR $<2$. The computed and measured VSWRs of the SsTF antenna are also presented in Fig. 2, and they are in good agreement. The deteriorations observed at lower frequencies in the figure for the basic antenna are sup- pressed, and the proposed SsTF antenna operates between 1.1 and $9.1 \mathrm{GHz}(8.3: 1)$. Additionally, the measured VSWR is lower than 2 between 9.4 and $12.9 \mathrm{GHz}$. Even though $2<\mathrm{VSWR}<$ 2.28 in a narrow band between 9.1 and $9.4 \mathrm{GHz}$, these deteriorations make the antenna unsuitable for UWB and X-band. To suppress them by providing a better impedance transition, the feedline is modified by employing a patch at only one side of the feeding point of the antenna, and its size is optimized as in [17] and [18], leading to the dimensions of $10 \mathrm{~mm} \times 3 \mathrm{~mm}$. The main information about similar transitions is given in [19]. The resulting antenna is called SsTF PLPDA antenna with a feeding point patch (SsTFwP) and it is shown in Fig. 1(c). Its computed and measured VSWR results are also presented in Fig. 2. As seen from the figure, SsTFwP antenna operates between 1.1 and $13.8 \mathrm{GHz}(12.5: 1)$ with VSWR $<2$ including UWB and $\mathrm{X}$-band. The computed and measured values are in good agreement especially at lower frequencies. When the results for SsTF and SsTFwP given in Fig. 2 are compared, it is also observed that the feeding point patch decreases and increases VSWR levels slightly above and below $8 \mathrm{GHz}$, respectively.

Since SsTFwP antenna has a superior bandwidth performance, the other fundamental and important antenna parameters - realized gain, radiation patterns, and group delay-are examined only for that one. Computed and measured peak realized gains are shown in Fig. 3. It is seen that they reach the desired value of $7 \mathrm{~dB}$ at some frequencies, and the average computed and measured peak realized gains are 5.5 and $5.2 \mathrm{~dB}$, respectively. The computed and the measured normalized coand cross-polarization patterns are presented in Fig. 4. The copolarized radiation patterns, especially the ones at lower frequencies, are in very good agreement. It is observed from the radiation patterns that the antenna is more directive at lower frequencies, therefore its front-to-back ratio is greater when compared to higher frequencies. The directivity of the antenna at higher frequencies might be improved by increasing the number of elements with realizable widths. In that case, the feedline and the patch must be optimized again to achieve good impedance matching. When the measured values are examined, 


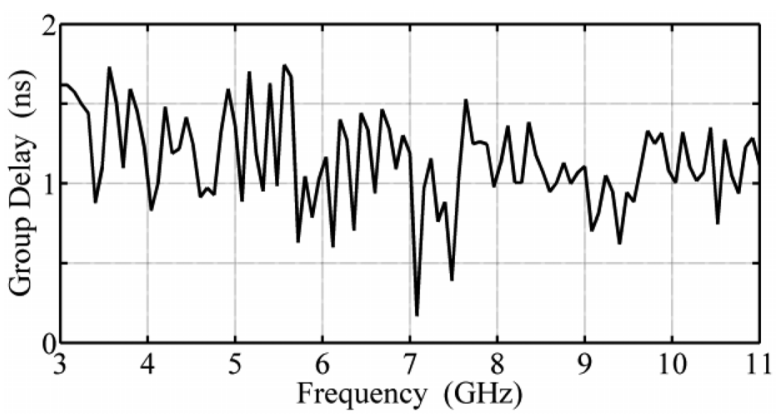

Fig. 5. Measured group delay of SsTFwP antenna.

it is seen that the cross-polarization discrimination levels are $20 \mathrm{~dB}$ or higher in the direction of maximum radiation at low frequencies, but these levels decrease at high frequencies. In the experimental study, Huber+Suhner SMA connectors were used and the 3-D geometric models of the connectors in STP format are provided in their Web site, but it was not possible to integrate the SMA connector and the coaxial cable into the simulations to take into account their influences, which might be the reason for the differences between the simulated and measured radiation patterns. In order to limit its influence, the coaxial cable is positioned perpendicular to the plane of the SsTFwP antenna. Group delay $\tau_{g}$ is used to examine the time delay of signal while transmitting through a device, and it is given by $\tau_{g}=-d \emptyset / d \omega$, where $\emptyset$ is the phase response of signal and $\omega$ is the angular frequency. Although constant group delay is desired to avoid signal distortions for perfect signal transmission, it is not generally possible in applications, and small deviations are acceptable. Since the group delay performance of the antenna has a critical role especially in impulse-based communication applications, measurements were made for only UWB. Group delay was obtained by using the method described in [20], and the antennas were placed $20 \mathrm{~cm}$ away from each other. The measured group delay of the SsTFwP antenna is shown in Fig. 5, and the antenna has a good performance with a maximum deviation of $1.5 \mathrm{~ns}$ in UWB.

\section{CONCLUSION}

In this study, two PLPDA antennas were designed by feeding line modifications with the least number of elements and their simplest form, narrow strips. The first proposed design was obtained by introducing a subsectional tapered feeding line, which is also the reduced form of the standard PLPDA presented in the literature. A rectangular patch was placed at the feeding point of the antenna to enhance its high frequency performance. The final proposed antenna has a superior bandwidth from 1.1 to $13.8 \mathrm{GHz}(12.5: 1)$ with overall perfect matching, and it is suitable for GPS (L1-L5), PCS, IMT-2000, Bluetooth, WLAN, WiMAX, UWB, and X-band with an average peak realized gain of $5.2 \mathrm{~dB}$. Its group delay performance is also satisfactory with a maximum of $1.5 \mathrm{~ns}$ deviation in the whole UWB. In addition, it has a very simple structure, and its fabrication and integration are very easy when compared to those of the other PLPDA antennas presented in the literature. As a result, it is a very good candidate for wideband, multipurpose, and portable devices.

\section{ACKNOWLEDGMENT}

The authors thank FIGES A.S. for supplying the ANSYS HFSS software license, and the reviewers for their constructive comments to improve this letter.

\section{REFERENCES}

[1] R. DuHamel and D. Isbell, "Broadband logarithmically periodic antenna structures," in IRE Int. Conv. Rec., 1957, pp. 119-128.

[2] G. Zhai, Y. Cheng, Q. Yin, S. Zhu, and J. Gao, "Gain enhancement of printed log-periodic dipole array antenna using director cell," IEEE Trans. Antennas Propag., vol. 62, no. 11, pp. 5915-5919, Nov. 2014.

[3] A. Chauloux, F. Colombel, M. Himdi, J. Lasserre, and P. Pouliguen, "Low return loss printed log periodic dipole antenna," IEEE Antennas Wireless Propag. Lett., vol. 13, pp. 503-506, 2014.

[4] D. Elsheakh and E. Abdallah, "Compact printed log-periodic dipole antenna for terrestrial digital video broadcast application," Microw. Opt. Technol. Lett., vol. 56, pp. 1002-1007, Apr. 2014.

[5] G. Casula, P. Maxia, G. Montisci, G. Mazzarella, and F. Gaudiomonte, "A printed LPDA fed by a coplanar waveguide for broadband applications," IEEE Antennas Wireless Propag. Lett., vol. 12, pp. 1232-1235, 2013.

[6] R. Kumari and S. K. Behera, "Wideband log-periodic dielectric resonator array with overlaid microstrip feed line," Microw., Antennas Propag., vol. 7, no. 7, pp. 582-587, May 2013.

[7] J. Yeo and J.-I. Lee, "Miniaturized LPDA antenna for portable direction finding applications," ETRI J., vol. 34, pp. 118-121, Feb. 2012.

[8] S. M. Hashemi, V. Nayyeri, M. Soleimani, and A.-R. Mallahzadeh, "Designing a compact-optimized planar dipole array antenna," IEEE Antennas Wireless Propag. Lett., vol. 10, pp. 243-246, 2011.

[9] D. E. Anagnostou, J. Papapolymerou, M. M. Tentzeris, and C. G. Christodoulou, "A printed log-periodic Koch-dipole array (LPKDA)," IEEE Antennas Wireless Propag. Lett., vol. 7, pp. 456-460, 2008.

[10] A. Asghar, M. Malick, M. Karlsson, and A. Hussain, "A multiwideband planar monopole antenna for 4G devices," Microw. Opt. Technol. Lett., vol. 55, pp. 589-593, Mar. 2013.

[11] P. Vainikainen et al., "More than 20 antenna elements in future mobile phones, threat or opportunity?," in Proc. 3rd Eur. Conf. Antennas Propag., Berlin, Germany, 2009, pp. 2940-2943.

[12] S. Praveen et al., "Miniature radar for mobile devices," in Proc. IEEE High Perform. Extreme Comput. Conf., 2013, pp. 1-8.

[13] L. Chang, J.-q. Zhang, M.-f. Zhang, Y.-f. Wang, and B.-m. Li, "Bandwidth improvement of a printed log-periodic antenna," IETE Tech. Rev., vol. 31, no. 34, pp. 266-270, Jul.-Aug. 2014.

[14] M. Mirzapour, S. Razavi, and S. M. Armaki, "Ultra-wideband planar LPDA antenna with mode converter balun," Electron. Lett., vol. 50, no. 12 , pp. 848-850, Jun. 2014.

[15] C. A. Balanis, Antenna Theory: Analysis and Design. New York, NY, USA: Wiley, 2012.

[16] C. Campbell, I. Traboulay, M. Suthers, and H. Kneve, "Design of stripline log-periodic dipole antenna," IEEE Trans. Antennas Propag., vol. AP-25, no. 5, pp. 718-721, Sep. 1977.

[17] B. G. Duffley, G. A. Morin, M. Mikavica, and Y. M. M. Antar, "A wide-band printed double-sided array," IEEE Trans. Antennas Propag., vol. 52, no. 2, pp. 628-631, Feb. 2004.

[18] M. Sonkki, E. Antonino-Daviu, M. Ferrando-Bataller, and E. T. Salonen, "Planar wideband polarization diversity antenna for mobile terminals," IEEE Antennas Wireless Propag. Lett., vol. 10, pp. 939-942, 2011.

[19] M. Gans, D. Kajfez, and V. H. Rumsey, "Frequency independent baluns," Proc. IEEE, vol. 53, no. 6, pp. 647-648, Jun. 1965.

[20] P. McEvoy, M. John, S. Curto, and M. J. Ammann, "Group delay performance of ultra wideband monopole antennas for communication applications," in Proc. Antennas Propag. Conf., Loughborough, U.K., 2008, pp. 377-380. 